\title{
On Ribaucour transformations and minimal surfaces
}

\author{
M. V. Lemes * K. Tenenblat ${ }^{\dagger}$
}

\begin{abstract}
Obtaining minimal surfaces by a Ribaucour transformation requires solving a system of partial differential equations, which is a Darboux transformation. We consider the system in complex variables for minimal surfaces parametrized by isothermal coordinates and lines of curvature. We relate the data of the Enneper-Weierstrass representation of minimal surfaces associated by Ribaucour transformations. The system of equations is solved explicitly for a special class of surfaces, which include important minimal surfaces. The class is characterized in terms of the Weierstrass data. The application of these results to the helicoid provides a new family of complete, minimal surfaces, of genus zero, immersed in $\mathbb{R}^{3}$, with an infinite number of embedded planar ends.
\end{abstract}

Key words: Minimal surfaces, Weierstrass representation, Ribaucour transformation, Darboux transformation.

\section{Introduction}

In the last two decades, the construction of new complete minimal surfaces in $\mathbb{R}^{3}$ has been a very active topic of research (see for instance $[\mathrm{CHM}],[\mathrm{Co}],[\mathrm{HM}]$ and $[\mathrm{JM}]$ ). The main tool in such constructions has been the Enneper-Weierstrass representation. Recently, the Ribaucour transformation was shown to be useful to provide new complete minimal surfaces (see [CFT2]).

Ribaucour transformations for hypersurfaces, parametrized by lines of curvature, were studied by Bianchi [Bi]. He showed that these transformations can be used to obtain surfaces of constant Gaussian curvature or minimal surfaces from a given such surface. Recently, Corro, Ferreira and Tenenblat, generalized the results of Bianchi to surfaces with any parametrization and they used Ribaucour transformations to associate Dupin hypersurfaces [CFT1] and linear Weingarten surfaces [CFT3].

Although the transformation for minimal surfaces is a classical result, it was applied, for the first time, to Enneper's surface and to the catenoid recently [CFT2]. New families of complete minimal surfaces were obtained, of genus zero, immersed in $\mathbb{R}^{3}$, with a finite or infinite number of planar ends and one or two nonplanar ends. Other results on Ribaucour transformations can be found in [CFT3], [CT] and [W].

*supported by $\mathrm{CNPq}$

†partially supported by $\mathrm{CNPq}$ 
Applying Ribaucour transformations to minimal surfaces corresponds to solving a linear system of partial differential equations. Although the system is integrable, finding its solutions may be difficult.

In this paper we consider minimal surfaces in $\mathbb{R}^{3}$, with no umbilic points, parametrized (without loss of generality) by isothermal coordinates and lines of curvature. For such coordinates, we rewrite the system of differential equations in complex variables and we relate the Weierstrass data of two minimal surfaces associated by a Ribaucour transformation. We explicitly solve the system of differential equations for a special class of minimal surfaces. Such surfaces are characterized in terms of the Enneper-Weierstrass representation. This class of surfaces includes, important minimal surfaces such as the Bonnet's minimal surfaces, the helicoid, the catenoid and Enneper's surface. We obtain new families of complete minimal surfaces by applying the theory to the helicoid.

The paper is organized as follows: in section 1, we consider basic facts of Ribaucour transformations for surfaces in $\mathbb{R}^{3}$. We recall the additional condition for such a transformation to associate minimal surfaces to a given minimal surface.

In section 2, we consider minimal surfaces parametrized by lines of curvature and isothermal coordinates. We rewrite the system of differential equations in complex variables and we relate the Enneper-Weierstrass data of the minimal surfaces associated by a Ribaucour transformation.

In section 3, we solve the system of differential equations for a special class of surfaces, whose conformal factor satisfies a certain condition. This condition is shown to be equivalent to requiring a special type of Weierstrass data (3.5). Moreover we restrict the theory of section 2 to this class of surfaces.

In section 4, we apply these results to the helicoid and we provide a new family of complete minimal surfaces of genus zero, immersed in $\mathbb{R}^{3}$, with infinitely many embedded planar ends. We also obtain the Enneper-Weierstrass representation of the minimal surfaces, associated by a Ribaucour transformations to the catenoid and to Enneper's surface.

Finally, we want to thank the referee, whose comments improved substantially the final version of this paper.

\section{Ribaucour transformations}

In this section, we recall some basic facts on Ribaucour transformations. An important result of this section, obtained by Corro-Ferreira-Tenenblat, states that the ends generated by a Ribaucour transformation, applied to a minimal surface, are embedded planar ends. For the proofs and more details, see [CFT1], [CFT2] and [CT].

Let $M$ and $\widetilde{M}$ be two orientable surfaces in $\mathbb{R}^{3}$, with Gauss maps $N$ and $\widetilde{N}$ respectively. Let $e_{1}, e_{2}$ be orthonormal principal vector fields on $M$. We say that $\widetilde{M}$ is associated to $M$ by a Ribaucour transformation with respect to $e_{1}, e_{2}$ if, and only if, there exist a differentiable function $h: M \rightarrow \mathbb{R}$ and a diffeomorphism $\psi: M \rightarrow \widetilde{M}$ such that

a) $p+h(p) N(p)=\psi(p)+h(p) \tilde{N}(\psi(p))$ for all $p \in M$,

b) the subset $p+h(p) N(p), p \in M$, is a surface, 
c) $d \psi\left(e_{1}\right)$ and $d \psi\left(e_{2}\right)$ are orthogonal principal directions on $\widetilde{M}$.

We observe that either side of the equality in $a)$ is the center of a sphere of radius $h(p)$, that is tangent to $M$ an $\widetilde{M}$ at $p$ and $\psi(p)$ respectively. When the radius $h$ tends to infinity when $p \rightarrow p_{0}$, one may have two possibilities: either the surface $\widetilde{M}$ extends nicely to the point $\psi\left(p_{0}\right)$ or else the new surface is not defined at that point. This is the case of the planar ends produced on minimal surfaces (see more comments later in this section). So one has to treat those points separately.

We say that $\widetilde{M}$ is locally associated to $M$ by a Ribaucour transformation with respect to $e_{1}, e_{2}$ if for any $p \in \widetilde{M}$ there exists a neighborhood $\widetilde{V}$ of $p$ in $\widetilde{M}$ and an open subset $V \subset M$, such that $\widetilde{V}$ is associated to $V$, by a Ribaucour transformation with respect to $e_{1}, e_{2}$. When the principal curvatures have multiplicity one, we do not need to refer to $e_{1}$, $e_{2}$ and we say simply that $\widetilde{M}$ is (locally) associated to $M$ by a Ribaucour transformation.

This is the revised version of the classical definition of a Ribaucour transformation, introduced in $[\mathrm{CT}]$. It allows to extend the transformation to the case when there is an open set of umbilic points. Actually, one shows in $[\mathrm{CT}]$ that generically any surface of $\mathbb{R}^{3}$ can be locally obtained by applying a Ribaucour transformation to an open subset of the plane or to an open subset of the sphere, as long as one chooses the appropriate frame $e_{1}$, $e_{2}$.

One can also show that, when the principal directions have multiplicities bigger than one, by choosing different vector fields $e_{1}, e_{2}$, one gets different associated surfaces by such a transformation (see Example 3.7 in $[\mathrm{CT}]$ ). It would be interesting to find out if Ribaucour transformations can be applied to a neighborhood of an isolated umbilic point.

The definition of Ribaucour transformation requires a diffeomorphism $\psi$. Examples, that have been treated by such transformations, show that one may have two complete surfaces in $\mathbb{R}^{3}$, which are not homeomorphic, although locally they are associated by Ribaucour transformations. See for example in [CFT2] the catenoid and the minimal surfaces associated to the catenoid, which are topologically either the sphere punctured at any finite number of points or the sphere punctured at an infinite numbers of points.

Obtaining the Ribaucour transformation of a surface in $\mathbb{R}^{3}$ corresponds to solving a second order nonlinear partial differential equation for $h$, where one needs $h$ not to be a radius of curvature (this is equivalent to condition (b) of the definition, see pg.144 [CFT1]). However, this equation can be linearized by considering $h=\Omega / W$. One can show the next result (see $[\mathrm{CFT} 1])$.

Proposition 1.1 Let $M$ be an orientable surface of $\mathbb{R}^{3}$. Assume that $e_{1}, e_{2}$ are orthonormal principal vector fields on $M,-\lambda_{1}$ and $-\lambda_{2}$ the corresponding principal curvatures, i.e., $d N\left(e_{i}\right)=\lambda_{i} e_{i}$. If a surface $\widetilde{M}$ is locally associated to $M$ by a Ribaucour transformation with respect to $e_{1}, e_{2}$ then on a simply connected domain $h=\Omega / W$, where $\Omega$ and $W$ are 
functions which satisfy

$$
\begin{aligned}
d \Omega_{i}\left(e_{j}\right) & =\Omega_{j} \omega_{i j}\left(e_{j}\right), \text { for } i \neq j, \\
d \Omega & =\sum_{i=1}^{2} \Omega_{i} \omega_{i}, \\
d W & =-\sum_{i=1}^{2} \Omega_{i} \lambda_{i} \omega_{i}
\end{aligned}
$$

where $\omega_{i}$ are the dual forms of $e_{i}$ and $\omega_{i j}$ is the connection form.

The next result describes the surfaces $\widetilde{M}$, associated to $M$ by a Ribaucour transformation in terms of the solutions of the system.

Theorem 1.2 Let $M$ be an orientable surface of $\mathbb{R}^{3}$ parametrized by $X: U \subset \mathbb{R}^{2} \rightarrow M$. Assume that $e_{i}, 1 \leq i \leq 2$ are orthogonal principal directions,$-\lambda_{i}$ the corresponding principal curvatures and $N$ is the Gauss map of $M$. A surface $\widetilde{M}$ is locally associated to $M$ by a Ribaucour transformation with respect to $e_{i}$ if, and only if, there exist differentiable functions $W, \Omega, \Omega_{1}, \Omega_{2}: V \subset U \rightarrow \mathbb{R}$, which satisfy the system (1.1) - (1.3), with

$$
W S\left(W+\lambda_{i} \Omega\right)\left(S-\Omega T^{i}\right) \neq 0 \quad i=1,2
$$

where

$$
\begin{aligned}
S & =\sum_{i=1}^{2}\left(\Omega_{i}\right)^{2}+W^{2}, \\
T^{i} & =2\left[d \Omega_{i}\left(e_{i}\right)+\sum_{k=1}^{2} \Omega_{k} \omega_{k i}\left(e_{i}\right)+W \lambda_{i}\right],
\end{aligned}
$$

and $\widetilde{X}: V \subset U \rightarrow \widetilde{M}$, is a parametrization of $\widetilde{M}$ given by

$$
\widetilde{X}=X-\frac{2 \Omega}{S}\left(\sum_{i=1}^{2} \Omega_{i} e_{i}-W N\right)
$$

Moreover, the normal map of $\widetilde{X}$ is given by

$$
\widetilde{N}=N+\frac{2 W}{S}\left(\sum_{i=1}^{2} \Omega_{i} e_{i}-W N\right) .
$$

In principle, Ribaucour transformations are local transformations determined by the solutions of the system of equations (1.1) - (1.3) defined on a simply connected domain. Even if the solution is globally defined on the universal covering of the surface $M$, a point where $h=\Omega / W$ tends to infinity may not correspond to any point on the associated surface. For example, this is how planar ends are produced on minimal surfaces by Ribaucour transformations (see Theorem 1.4). 
A proof of Theorem 1.2 can be found in [CFT2] (see also [CT]). One can also show that the parametrization $\widetilde{X}$ given by (1.6) may extend regularly to points where $W\left(W+\lambda_{i} \Omega\right)=$ 0 , whenever $S\left(S-\Omega T^{i}\right) \neq 0$. From now on, whenever we say that a surface $\widetilde{M}$ is locally associated to $M$ by a Ribaucour transformation, we are assuming that there are differentiable functions $\Omega_{i}, \Omega$ and $W$, locally defined, satisfying the system (1.1) - (1.3) and $S\left(S-\Omega T^{i}\right) \neq 0, i=1,2$.

Bianchi [Bi] showed that, by requiring an additional algebraic condition

$$
\Omega_{1}^{2}+\Omega_{2}^{2}+W^{2}=2 c \Omega W
$$

on the solution of the system $(1.1)-(1.3)$, Ribaucour transformations can be used as a method of constructing minimal surfaces. This is essentially the content of the next theorem.

Theorem 1.3 Let $M$ be an orientable minimal surface of $\mathbb{R}^{3}$, with no umbilic points, parametrized by $X: V \subset \mathbb{R}^{2} \rightarrow M \subset \mathbb{R}^{3}$. Let $e_{1}, e_{2}$ be orthonormal principal vector fields and $-\lambda_{1},-\lambda_{2}$ the corresponding principal curvatures. Then, for any real constant $c \neq 0$, the system of equations

$$
\begin{aligned}
d \Omega_{i} & =\Omega_{j} \omega_{i j}+\left[c W+(W-c \Omega) \lambda_{i}\right] \omega_{i}, \quad i \neq j \\
d \Omega & =\sum_{i=1}^{2} \Omega_{i} \omega_{i} \\
d W & =-\sum_{i=1}^{2} \lambda_{i} \Omega_{i} \omega_{i}
\end{aligned}
$$

is integrable. Moreover, any solution of this system satisfies (1.8), on a simply connected domain, if the given initial conditions satisfy (1.8). In this case, the surface $\widetilde{X}$, associated to $X$ by a Ribaucour transformation, is a minimal surface, defined wherever $S\left(S-\Omega T^{i}\right) \neq$ 0 and it is given by

$$
\widetilde{X}=X-\frac{1}{c W}\left(\Omega_{1} e_{1}+\Omega_{2} e_{2}-W N\right)
$$

and its normal map $\widetilde{N}$ by

$$
\widetilde{N}=N+\frac{1}{c \Omega}\left(\Omega_{1} e_{1}+\Omega_{2} e_{2}-W N\right) .
$$

We observe that (1.8), with the condition that $\Omega_{i} \neq 0$, defines $d \Omega_{i}\left(e_{i}\right)$ as in (1.9) (see the proof of Theorem 1.7 in [CFT2]), while (1.9) implies (1.8), as long as the initial conditions for $\Omega_{1}, \Omega_{2}, \Omega$ and $W$ satisfy (1.8).

In section 4 , we will need the following result on the points that annihilate $S$, where $S$ is given by (1.5). Such a point $p_{0}$, generically, produces a planar embedded end on the surface $\widetilde{X}$. Moreover, the behavior of $\widetilde{X}$ in a neighborhood of $p_{0}$ is also described.

Theorem 1.4 (Corro - Ferreira - Tenenblat) Let $\tilde{X}: D \backslash\left\{p_{0}\right\} \rightarrow \mathbb{R}^{3}$ be a minimal surface, locally associated by a Ribaucour transformation to a minimal surface $X: D \rightarrow \mathbb{R}^{3}$ such that, the functions $\Omega_{i}, \Omega$ and $W$ are defined on $D$. Let $\widetilde{N}$ and $N$ be the normal maps of $\widetilde{X}$ and $X$, respectively. If $S\left(p_{0}\right)=0, \Omega\left(p_{0}\right) \neq 0$ and $S(p) \neq 0$ for all $p \in D \backslash\left\{p_{0}\right\}$, then 
(a) for any divergent curve $\gamma:[0,1) \rightarrow D \backslash\left\{p_{0}\right\}$ such that $\lim _{t \rightarrow 1} \gamma(t)=p_{0}$ the length of $\tilde{X}(\gamma)$ is infinite.

(b) The minimal surface $\widetilde{X}$ has an embedded planar end at $p_{0}$, and $\lim _{p \rightarrow p_{0}} \tilde{N}(p)=N\left(p_{0}\right)$.

The proof of this theorem can be found in [CFT2], Proposition 1.8.

We conclude this section by observing that the Ribaucour transformation of a minimal surface, given in Theorem 1.3, is in fact a Darboux transformation (i.e. it transforms an isothermic minimal surface into such a surface). This property was proved, although not stated as a theorem, in [CFT2].

Theorem 1.5 (Corro - Ferreira - Tenenblat) The Ribaucour transformation of a minimal surface given in Theorem 1.3 is a Darboux transformation. Let $X(z, \bar{z})$ be a local parametrization of a minimal surface such that the fundamental forms are $I=\varphi^{2}\left(d x^{2}+d y^{2}\right)$ and $I I=\ell\left(d x^{2}-d y^{2}\right), \ell \neq 0, \ell \in \mathbb{R}$. If $\widetilde{X}(x, y)$ is a minimal surface associated to $X$ by a Ribaucour transformation, then the fundamental forms of $\widetilde{X}$ are given by $\widetilde{I}=\widetilde{\varphi}^{2}\left(d x^{2}+d y^{2}\right)$ and $\widetilde{I I}=\ell\left(d x^{2}-d y^{2}\right)$ where

$$
\widetilde{\varphi}=\left|\frac{\ell \Omega}{\varphi W}\right|
$$

and $\Omega, W$ is a solution of (1.9), with a given initial condition satisfying (1.8).

This result was obtained in the proof of Proposition 1.8 of [CFT2]. The expression (1.12) also follows immediately by considering the formula (39) of [CFT3], with $H=0$.

It would be interesting to relate the Ribaucour transformation of Theorem 1.3 with the minimal Darboux transformation. In general, starting with a minimal surface one gets a 4-real parameter ( 4 initial conditions for (1.9) and $c \neq 0$, related by the condition (1.8)) family of minimal surfaces associated by Ribaucour transformations. This fact suggests that this transformation may provide a larger family of minimal surfaces than the minimal Darboux transformations.

\section{A different approach}

As we have seen in the previous section, applying Ribaucour transformations to minimal surfaces corresponds to solving the system of equations (1.1) - (1.3). Although this is an integrable system of differential equations, finding its solutions may be difficult.

In this section, we consider minimal surfaces in $\mathbb{R}^{3}$, parametrized by isothermal coordinates and lines of curvature. For such coordinates, we rewrite the system of differential equations in complex variables. Moreover, we relate the Enneper-Weierstrass data of two minimal surfaces associated by a Ribaucour transformation.

Let $M$ be a minimal surface with no umbilic points. Without loss of generality, we may assume that $M$ has an isothermal parametrization $X(z, \bar{z})$, by lines of curvature, whose quadratic forms are given by

$$
I=\varphi^{2} d z d \bar{z} \quad \text { and } \quad I I=-\frac{1}{2}\left(d z^{2}+d \bar{z}^{2}\right)
$$


where $z=x+i y \in U \subset \mathbb{C}$.

The data in the Enneper-Weierstrass representation of a minimal surface in such coordinates is a meromorphic function $g(z)$ and $f=1 / g^{\prime}$. The conformal factor $\varphi$ and the function $g$ are related on a simply connected domain by

$$
\varphi=\frac{1+|g|^{2}}{2\left|g^{\prime}\right|} .
$$

Observe that $g$ and its transformations $\frac{a g-b}{\bar{b} g+\bar{a}},|a|^{2}+\left|b^{2}\right|>0$, provide all possibilities for (2.2) to hold.

A straightforward computation provides a system of differential equations equivalent to $(1.1)-(1.3)$, given by the following result.

Proposition 2.1 Let $M$ be an orientable minimal surface of $\mathbb{R}^{3}$, without umbilic points, parametrized by $X(z, \bar{z})$ such that its quadratic forms are given by (2.1). Then the system of equations (1.1) - (1.3) is equivalent to the integrable system

$$
\begin{aligned}
\Omega_{z z} & =\frac{1}{2}(W-c \Omega)+2 \Omega_{z} \frac{\varphi_{z}}{\varphi}, \\
\Omega_{z \bar{z}} & =\frac{c \varphi^{2} W}{2} \\
W_{z} & =-\frac{\Omega_{\bar{z}}}{\varphi^{2}}
\end{aligned}
$$

with $0 \neq c \in \mathbb{R}$ and the initial conditions satisfying

$$
\left(4 W_{z} W_{\bar{z}}+W^{2}-2 c \Omega W\right)\left(z_{0}\right)=0, \quad \text { for some } z_{0} \in U .
$$

Any solution of (2.3)-(2.5) with (2.6), defined on a simply connected domain, satisfies (2.6) for all $z \in U$. Moreover the surface $\widetilde{X}$, associated to $X$ is a minimal surface parametrized by

$$
\widetilde{X}=X+\frac{2}{c W}\left(W_{z} X_{z}+W_{\bar{z}} X_{\bar{z}}\right)+\frac{1}{c} N
$$

and its normal map is given by

$$
\widetilde{N}=\left(1-\frac{W}{c \Omega}\right) N-\frac{2}{c \Omega}\left(W_{z} X_{z}+W_{\bar{z}} X_{\bar{z}}\right) .
$$

We will now show the relation between the meromorphic functions $g(z)$ and $\widetilde{g}(z)$ of minimal surfaces associated by a Ribaucour transformation

Proposition 2.2 Let $g(z)$ and $f=1 / g^{\prime}$, be the data of a minimal surface $M$ in $\mathbb{R}^{3}$, in the Enneper-Weierstrass representation. Then the system of equations $(2.3)-(2.5)$, where $c \neq 0$ and $\varphi$ is given by (2.2), is integrable. Any solution $\Omega, W$ of this system defined on a simply connected domain, with initial condition satisfying (2.6), determines a minimal 
surface $\widetilde{M}$, locally associated to $M$ by a Ribaucour transformation, whose Weierstrass data is given by

$$
\widetilde{g}(z)=\frac{2 g(c \Omega-W)+\left(|g|^{2}+1\right)\left(W_{z} f g^{2}-W_{\bar{z}} \bar{f}\right)}{2 c \Omega+W\left(|g|^{2}-1\right)+\left(|g|^{2}+1\right)\left(W_{z} f g+W_{\bar{z}} \bar{f} \bar{g}\right)}
$$

and $\widetilde{f}=1 / \widetilde{g}^{\prime}$.

Proof. Let $X(z, \bar{z})$ be a local parametrization of the minimal surface $M$, whose quadratic forms are given by (2.1). Then, in terms of the $g$ and $f$, the normal map of $M$ is

$$
N=\left(\frac{2 \operatorname{Re} g}{|g|^{2}+1}, \frac{2 \operatorname{Im} g}{|g|^{2}+1}, \frac{|g|^{2}-1}{|g|^{2}+1}\right)
$$

and

$$
X_{z}=\left(\frac{1}{4} f\left(1-g^{2}\right), \frac{i}{4} f\left(1+g^{2}\right), \frac{f g}{2}\right) .
$$

Therefore, it follows from Proposition 2.1 and Theorem 1.5, that the expression $\widetilde{X}(z, \bar{z})$, given by (2.7) parametrizes a minimal surface, whose fundamental forms satisfy (2.1). From (2.8), we have

$$
\begin{aligned}
\frac{|\widetilde{g}|^{2}-1}{|\widetilde{g}|^{2}+1} & =\left(1-\frac{W}{c \Omega}\right) \frac{|g|^{2}-1}{|g|^{2}+1}-\frac{2}{c \Omega} \operatorname{Re}\left(W_{z} f g\right), \\
\frac{2 \operatorname{Re} \widetilde{g}}{|\widetilde{g}|^{2}+1} & =\left(1-\frac{W}{c \Omega}\right) \frac{2 \operatorname{Re} g}{|g|^{2}+1}-\frac{1}{c \Omega} \operatorname{Re}\left(W_{z} f\left(1-g^{2}\right)\right), \\
\frac{2 \operatorname{Im} \widetilde{g}}{|\widetilde{g}|^{2}+1} & =\left(1-\frac{W}{c \Omega}\right) \frac{2 \operatorname{Im} g}{|g|^{2}+1}-\frac{1}{c \Omega} \operatorname{Re}\left(i W_{z} f\left(1+g^{2}\right)\right) .
\end{aligned}
$$

From the first equation we get

$$
|\widetilde{g}|^{2}+1=\frac{2 c \Omega\left(|g|^{2}+1\right)}{2 c \Omega+W\left(|g|^{2}-1\right)+\left(|g|^{2}+1\right)\left(W_{z} f g+W_{\bar{z}} \bar{f} \bar{g}\right)}
$$

and from the other two equations we have

$$
2 \widetilde{g}=\left(|\widetilde{g}|^{2}+1\right)\left\{\left(1-\frac{W}{c \Omega}\right) \frac{2 g}{|g|^{2}+1}+\frac{1}{c \Omega}\left(W_{z} f g^{2}-W_{\bar{z}} \bar{f}\right)\right\} .
$$

The last two equations prove (2.9) .

\section{A special class of surfaces}

In this section, we will consider a special class of surfaces in $\mathbb{R}^{3}$, parametrized by isothermal coordinates, whose conformal factor $\varphi$ satisfies the condition

$$
\varphi_{z z}=A^{2} \varphi, \quad A \in \mathbb{C} .
$$


We will show that this condition implies that $K \varphi^{4}$ is a real constant, where $K$ is the Gaussian curvature. By considering the minimal surfaces which belong to this special class of surfaces, we will characterize such surfaces in terms of the meromorphic function $g(z)$ of the Enneper-Weierstrass representation. One can see that Enneper's surface, the catenoid, the helicoid and the Bonnet's minimal surfaces (all lines of curvatures are planar) belong to this class of surfaces. Moreover, we will provide explicitly all solutions of $(2.3)-(2.6)$ and we will restrict the theory of the previous section to this class of surfaces.

We start observing that $\varphi$ satisfies (3.1) if, and only if,

$$
\varphi= \begin{cases}a_{1} e^{2 \operatorname{Re} A z}+a_{2} e^{-2 \operatorname{Re} A z}+a_{3} e^{2 i \operatorname{Im} A z}+\bar{a}_{3} e^{-2 i \operatorname{Im} A z}, & \text { if } A \neq 0 \\ a_{1}|z|^{2}+a_{3} z+\bar{a}_{3} \bar{z}+a_{2}, & \text { if } A=0\end{cases}
$$

where $a_{1}, a_{2} \in \mathbb{R}, a_{3} \in \mathbb{C}$.

Proposition 3.1 Let $X(z, \bar{z})$ be a surface parametrized by isothermal coordinates, i.e., $I=\varphi^{2} d z d \bar{z}$. Assume $\varphi$ satisfies (3.1). Then $K \varphi^{4}$ is a real constant. If $X$ is a minimal surface whose fundamental forms are given by (2.1), then $\varphi$ is given by (3.2), where

$$
a_{1} a_{2}-\left|a_{3}\right|^{2}= \begin{cases}\frac{1}{16|A|^{2}}, & \text { if } A \neq 0, \\ \frac{1}{4}, & \text { if } A=0\end{cases}
$$

where, without loss of generality, we may assume that $a_{1}>0$. Moreover, $\varphi$ satisfies

$$
4\left(\varphi \varphi_{z \bar{z}}-\varphi_{z} \varphi_{\bar{z}}\right)=1 .
$$

In that case, the Weierstrass data of the minimal surface is globally given by

$$
g(z)= \begin{cases}4|A|\left(a_{1} e^{2 A z}+\bar{a}_{3}\right), & \text { if } A \neq 0, \\ 2\left(a_{1} z+\bar{a}_{3}\right), & \text { if } A=0, z \in \mathbb{C} .\end{cases}
$$

Proof. Since $X$ is parametrized by isothermal coordinates, the Gaussian curvature is given by

$$
K=-\frac{4}{\varphi^{4}}\left(\varphi_{z \bar{z}} \varphi-\varphi_{\bar{z}} \varphi_{z}\right) .
$$

Equation (3.1), implies that $\varphi$ is given by (3.2) and hence

$$
K \varphi^{4}= \begin{cases}-16|A|^{2}\left(a_{1} a_{2}-\left|a_{3}\right|^{2}\right), & \text { if } A \neq 0 \\ -4\left(a_{1} a_{2}-\left|a_{3}\right|^{2}\right), & \text { if } A=0\end{cases}
$$

For the minimal surface $X$, the principal curvatures are $\pm 1 / \varphi^{2}$, which implies that (3.3) and (3.4) hold. A straightforward computation shows that $g(z)$, given by (3.5), satisfies (2.2). This concludes the proof. 
Proposition 3.2 Let $\varphi(z)$ be a differential function that satisfies (3.2), (3.3) and (3.4). Then $\Omega, W$ is a solution of $(2.3)-(2.6)$ if, and only if,

$$
\begin{aligned}
\Omega & =\frac{1}{c}\left[2 \varphi^{2}\left(\frac{F}{\varphi}\right)_{z \bar{z}}+\frac{F}{\varphi}\right], \\
W & =\frac{F}{\varphi},
\end{aligned}
$$

where

$$
\begin{gathered}
F=\left\{\begin{array}{lc}
b_{1} e^{\operatorname{Re} \beta z}+b_{2} e^{-\operatorname{Re} \beta z}+b_{3} e^{i \operatorname{Im} \beta z}+\bar{b}_{3} e^{-i \operatorname{Im} \beta z}, & \text { if } \beta \neq 0, \\
b_{1} z \bar{z}+b_{3} z+\bar{b}_{3} \bar{z}+b_{2}, & \text { if } \beta=0,
\end{array}\right. \\
\beta=2 \rho \sqrt{A^{2}-\frac{c}{2}}, \quad \rho= \pm 1,
\end{gathered}
$$

$b_{1}, b_{2} \in \mathbb{R}, b_{3} \in \mathbb{C}$ and $b_{1} b_{2}-\left|b_{3}\right|^{2}=0$.

Proof. From (2.4) and (2.5) we have

$$
W_{z z}=-\frac{c}{2} W-2 W_{z} \frac{\varphi_{z}}{\varphi} .
$$

We consider $F=\varphi W$. Then it follows from (3.1) and (3.11) that

$$
F_{z z}=\frac{\beta^{2}}{4} F
$$

where $\beta$ is given by (3.10). Therefore $F$ satisfies (3.9). Hence $W=F / \varphi$, where $\varphi$ is given by (3.2) and (3.3). In order to conclude the proof, we observe that from (2.5) we have

$$
\Omega_{z}=-\varphi^{2}\left(\frac{F}{\varphi}\right)_{\bar{z}} .
$$

Hence

$$
\Omega_{z z}=-\varphi^{2}\left(\frac{F}{\varphi}\right)_{\bar{z} z}-2 \varphi \varphi_{z}\left(\frac{F}{\varphi}\right)_{\bar{z}} .
$$

Since $\Omega$ has to satisfy (2.3), using (3.4), (3.13) and (3.14) we conclude that $\Omega$ is given by (3.7) . Moreover, it follows from Proposition 2.1 that the identity

$$
4 \Omega_{z} \Omega_{\bar{z}}+F^{2}=2 c \varphi \Omega F
$$

holds. From this equation, we conclude that

$$
\varphi^{2}\left(F F_{z \bar{z}}-F_{z} F_{\bar{z}}\right)=F^{2}\left(\varphi \varphi_{z \bar{z}}-\varphi_{z} \varphi_{\bar{z}}-1 / 4\right) .
$$

Since $\varphi$ satisfies $(3.4)$, we have

$$
F F_{z \bar{z}}-F_{z} F_{\bar{z}}=0
$$

which is equivalent to saying that $b_{1} b_{2}-\left|b_{3}\right|^{2}=0$.

Conversely, it is a straightforward computation to verify that $\Omega$ and $W$, that are given respectively by (3.7) and (3.8), satisfy equations $(2.3)-(2.5)$ and (2.6) for all $z \in \mathbb{C}$. 
Remark 3.3 The function $F$ given by (3.9), when $\beta \neq 0$, can be rewritten as

$$
F(z, \bar{z})= \begin{cases}2\left|b_{3}\right|(\sigma \cosh (\operatorname{Re} \beta z+\mu)+\sin (\operatorname{Im} \beta z+\nu)) & \text { if }\left|b_{3}\right|>0, \sigma= \pm 1, \\ b_{1} e^{\operatorname{Re} \beta z} & \text { if }\left|b_{3}\right|=b_{2}=0, \\ b_{2} e^{-\operatorname{Re} \beta z} & \text { if }\left|b_{3}\right|=b_{1}=0 .\end{cases}
$$

Theorem 3.4 Let $M$ be an orientable minimal surface of $\mathbb{R}^{3}$, with no umbilic points, parametrized by $X(z, \bar{z})$ such that (2.1) holds. If the conformal factor satisfies (3.1), then

$$
\widetilde{X}=X+\frac{4}{c} \operatorname{Re}\left[\left(\ln \frac{F}{\varphi}\right)_{z} X_{z}\right]+\frac{1}{c} N
$$

is a three-parameter family of minimal surfaces defined on $\{z \in \mathbb{C} ; F(z) \neq 0\}$, locally associated to $X$ by a Ribaucour transformation, where $c \neq 0$ and $\varphi, F$ are given respectively by (3.2), (3.3) and (3.9).

If $g(z)$ and $f=1 / g^{\prime}$ are the data in the Enneper-Weierstrass representation of $M$, then $\widetilde{g}$, given by

$$
\widetilde{g}=\frac{2 g \varphi\left(\frac{F}{\varphi}\right)_{z \bar{z}}+\left|g^{\prime}\right|\left[\left(\frac{F}{\varphi}\right)_{z} f g^{2}+\left(\frac{F}{\varphi}\right)_{\bar{z}} \bar{f}\right]}{2 \varphi\left(\frac{F}{\varphi}\right)_{z \bar{z}}+\left|g^{\prime}\right|\left[\frac{F}{\varphi}+\left(\frac{F}{\varphi}\right)_{z} f g+\left(\frac{F}{\varphi}\right)_{\bar{z}} \bar{f} \bar{g}\right]},
$$

and $\tilde{f}=1 / \widetilde{g}^{\prime}$ provide a representation for $\widetilde{X}$.

Proof. The parametrization (3.17) follows from Propositions 2.1, 3.1 and 3.2.

We observe that, due to equations (3.7), (3.13) and (3.15) the initial conditions $\Omega\left(z_{0}\right)$, $\Omega_{z}\left(z_{0}\right), \Omega_{\bar{z}}\left(z_{0}\right)$ and $W\left(z_{0}\right)$ of $(2.3)-(2.5)$ are determined by choosing $F\left(z_{0}\right), F_{z}\left(z_{0}\right)$ and $F_{\bar{z}}\left(z_{0}\right)$. This gives three real parameters which together with parameter $c$ must satisfy (2.6). Therefore, $\widetilde{X}$ is a three-parameter family.

The expression of $\widetilde{g}$ given by (3.18) follows from Propositions 2.2 and 3.2.

Remark 3.5 It follows from equations (1.12), (3.4), (3.15) and from Proposition 3.2 that the fundamental forms of $\widetilde{X}$, given by (3.17), are of the form $\widetilde{I}=\widetilde{\varphi} d z d \bar{z}$ and $\widetilde{I I}=-\frac{1}{2}\left(d z^{2}+d \bar{z}^{2}\right)$ where

$$
\widetilde{\varphi}=\frac{\varphi}{2|c|}\left(4\left|\frac{F_{z}}{F}-\frac{\varphi_{z}}{\varphi}\right|^{2}+\frac{1}{\varphi^{2}}\right)
$$

\section{Applications}

In the previous section, we solved the system of differential equations which provides minimal surfaces locally associated by Ribaucour transformation to any surface of a special 
class of minimal surfaces. One can see that Enneper's surface, the catenoid and the helicoid are surfaces which belong to this class os surfaces.

In [CFT2], one can find the properties (number of ends, completeness, total curvature, symmetries) of the minimal surfaces associated to Enneper's surface and to the catenoid, according to the value of $c$, the constant of the algebraic condition (1.8).

In this section, we provide similar results for the helicoid, we obtain a new family of minimal surfaces of genus zero, immersed in $\mathbb{R}^{3}$. Moreover, we show that such surfaces are complete, have an infinite number of planar ends and have infinite total curvature. We conclude this section providing a Weierstrass representation for the minimal surfaces locally associated by Ribaucour transformations to Enneper's surface, to the catenoid and to the helicoid.

We observe that the usual Weierstrass representation of the helicoid, given by $f(z)=e^{-z}$ and $g(z)=-i e^{z}$, does not determine a parametrization by lines of curvature. In order to apply the theory of section 3 in the following proposition we will consider another Weierstrass representation for the helicoid.

Proposition 4.1 Let $X(z, \bar{z})$ be a parametrization of the helicoid given by the Weierstrass data $g(z)=-i e^{\sqrt{i} z}$ and $f=1 / g^{\prime}$, where $\sqrt{i}=e^{i \pi / 4}$. Excluding the helicoid, a minimal surface is locally associated to $X$ by a Ribaucour transformation as in Theorem 1.3 if, and only if, it belongs to a three-parameter family of minimal surfaces given by (3.17), where $c \neq 0$ is a real constant, $\varphi=\cosh (\operatorname{Re} \sqrt{i} z)$ and

$$
F(z, \bar{z})=\sigma \cosh (\operatorname{Re} \beta z+\mu)+\sin (\operatorname{Im} \beta z+\nu)
$$

for $\mu, \nu \in \mathbb{R}, \sigma= \pm 1$ and

$$
\beta=\rho \sqrt{i-2 c}, \quad \rho= \pm 1 \text {. }
$$

Any surface of the family $\widetilde{X}(z, \bar{z})$ is defined for $z \in \mathbb{C} \backslash\left\{z_{k}\right\}$, where

$$
z_{k}=\frac{-\rho \sqrt{2} m^{2}}{1+m^{4}}\left[\left(m-\frac{i}{m}\right) \mu+\left(\frac{1}{m}+i m\right)\left(\nu+\sigma \frac{\pi}{2}-2 k \pi\right)\right]
$$

for $k \in \mathbb{Z}$, and

$$
m=\sqrt{-2 c+\sqrt{4 c^{2}+1}}
$$

Proof. The first and second fundamental forms of $X$ are given by (2.1), where $\varphi=\cosh (\operatorname{Re} \sqrt{i} z)$. Moreover, it is easy to see that, $\varphi$ satisfies $(3.1)$, for $A=\sqrt{i} / 2$. Hence, the helicoid satisfies the hypothesis of Theorem 3.4. Thus, $\widetilde{X}$ is given by (3.17). Therefore, it follows from $\beta \neq 0$ and Theorem 3.4, that $F$ is given by (3.16), where $b_{1} b_{2}-\left|b_{3}\right|^{2}=0$.

If $b_{1} b_{2}=\left|b_{3}\right|=0$, then $F=e^{ \pm \operatorname{Re} \beta z}$. In this case, one can show that there exists a real constant $a \neq 0$, such that $\tilde{\varphi}(z)=\varphi(z+\sqrt{-i} a)$. Moreover, due to (1.12) the function $h=\frac{\Omega}{W}=\varphi \widetilde{\varphi} \neq 0$. It follows that $\widetilde{X}$ is the helicoid.

If $b_{1} b_{2}=\left|b_{3}\right| \neq 0$, without loss of generality (see (3.17)), we can choose $b_{1} b_{2}=\left|b_{3}\right|=1 / 2$ and we obtain (4.1).

Since $X(z, \bar{z})$ is defined for all $z \in \mathbb{C}$, it follows from $(3.17)$ that $\widetilde{X}(z, \bar{z})$ is defined for all $z \in \mathbb{C} \backslash\left\{z_{k} \mid F\left(z_{k}, \bar{z}_{k}\right)=0\right\}$, which implies that $\widetilde{X}$ is defined on $\mathbb{C}$ punctured at the points $z_{k}$ satisfying (4.3). 
In the following result we describe the ends of the minimal surfaces locally associated to the helicoid by a Ribaucour transformation.

Proposition 4.2 Any minimal surface $\widetilde{X}(z, \bar{z})$, defined by (3.17) for $z \in \mathbb{C} \backslash\left\{z_{k}\right\}$, has an infinite number of planar embedded ends $z_{k}$, given by (4.3). The Gauss map $\widetilde{N}$ of $\widetilde{X}$ satisfies,

$$
\lim _{z \rightarrow z_{k}} \tilde{N}(z)=N\left(z_{k}, \bar{z}_{k}\right)
$$

where $N$ is the Gauss map of $X$.

Proof. The result is a consequence of Theorem 1.4.

Remark 4.3 Considering the ends $z_{k}$ of any surface of the family $\widetilde{X}$ as points of $\mathbb{R}^{2}$, we conclude that all points $z_{k}=\left(x_{k}, y_{k}\right)$, given by (4.3), are on the intersection of the straight line

$$
y=m^{2} x+\sigma \rho \sqrt{2} m \mu
$$

with the straight lines

$$
y=\frac{-1}{m^{2}} x+\frac{\rho \sqrt{2}}{m}\left(\nu+\sigma \frac{\pi}{2}-2 k \pi\right), \quad k \in \mathbb{Z} .
$$

Corollary 4.4 Any surface locally associated by a Ribaucour transformation to the helicoid has infinite total curvature.

Proof. In Proposition 4.1 we saw that, excluding the helicoid, the minimal surfaces associated to the helicoid by a Ribaucour transformation are defined on $\mathbb{C} \backslash\left\{z_{k}\right\}$, where the points $z_{k}$ are given by (4.3). Each minimal surface corresponds to an immersion of the sphere punctured at a pole and at the points corresponding to the points $z_{k}$. Since the points $z_{k}$ are on a line of $\mathbb{R}^{2}$, then the corresponding points are on a circle on the sphere and accumulate at the pole. Therefore, any surface of the family $\widetilde{X}$ corresponds to an immersion of the unit sphere punctured at an infinite number of points. Therefore, it follows from Huber's Theorem (see [HUl) that the surfaces have infinite total curvature.

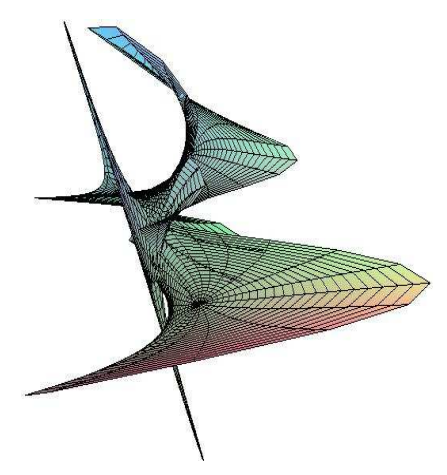

Figure 1: Minimal surface associated to helicoid in a neighborhood of the end $z_{0}$, with $c=\rho=\sigma=1$ and $\mu=\nu=0$. 

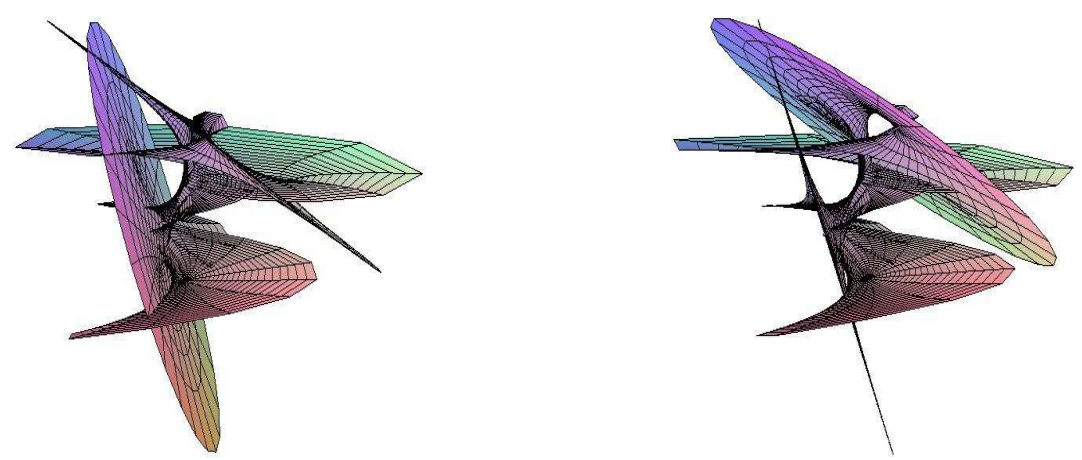

Figure 2: Minimal surface associated to helicoid in a neighborhood of the ends $z_{0}$ and $z_{1}$ with $c=\rho=\sigma=1$ and $\mu=\nu=0$.

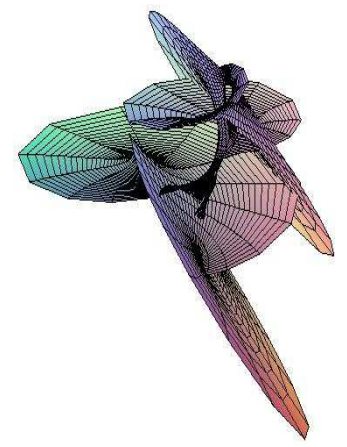

Figure 3: Minimal surface associated to helicoid in a neighborhood of the ends $z_{0}$ and $z_{1}$ with $c=\rho=\sigma=1, \mu=0$ and $\nu=\pi / 2$.

The illustrations above show examples of the minimal surfaces associated to the helicoid by a Ribaucour transformation. In Figures 1 and 2 we chose $c=\rho=\sigma=1$ and $\mu=\nu=0$ and in Figure 3 we chose $c=\rho=\sigma=1, \mu=0$ and $\nu=\pi / 2$. Moreover, we observe that the figures were obtained by considering polar coordinates on neighborhoods of the points $z_{0}$ and $z_{1}$, which generate two planar ends.

The surfaces locally associated to the catenoid and to Enneper's surface by a Ribaucour transformation are complete, as it was proved in [CFT2]. We will now show that the surfaces, associated to the helicoid by a Ribaucour transformation, are also complete.

In order to do so, we need to show that any divergent curve on the surface $\widetilde{X}$ has infinite length. Considering that $\widetilde{X}$ is defined on $\mathbb{C} \backslash\left\{z_{k}\right\}_{k \in \mathbb{Z}}$, it follows from Remark 4.3 that any divergent curve $\alpha(t)=(x(t), y(t))$ satisfies one of the following conditions when $t \rightarrow \infty: a)(x(t), y(t)) \rightarrow z_{k}$ for some $\left.k ; b\right) x^{2}+y^{2} \rightarrow \infty$ and for $t$ sufficiently large $(x(t), y(t)) \in \mathbb{C} \backslash T$, where $T$ is an open strip that contains the straight line $(4.5) ; c)$ $x^{2}+y^{2} \rightarrow \infty$ and $\forall t_{0} \exists t \geq t_{0}$ such that $\alpha(t) \in T$. We will show that a divergent curve satisfying any of the above conditions has infinite length, by adapting to our case the arguments used in [CFT2]. 
Lemma 4.5 Let $U$ be a subset of $\mathbb{R}^{2}$ endowed with a conformal metric

$$
d s^{2}=\widetilde{\varphi}^{2}(x, y)\left(d x^{2}+d y^{2}\right) .
$$

Assume that there are $a, b, r, \delta \in \mathbb{R}$, with $\delta>0$, such that the strip

$$
D=\left\{(x, y) \in \mathbb{R}^{2} \mid-\delta \leq y-a x-b \leq \delta\right\}
$$

is contained in $U$ and $\widetilde{\varphi}(x, y) \geq r>0$, for all $(x, y) \in D$. Then the length of any regular curve $\alpha$ joining two points in distinct components of $\mathbb{R}^{2} \backslash D$ satisfies

$$
\ell(\alpha) \geq \frac{2 r \delta}{\sqrt{1+a^{2}}}
$$

Proof. The line $R_{1}: a y+x=0$ is orthogonal to $D$ and $R_{1} \cap \partial D=\left\{P_{1}, P_{2}\right\}$ hence

$$
\left|P_{1}-P_{2}\right|=\frac{2 \delta}{\sqrt{1+a^{2}}}
$$

Setting $D^{+}=\left\{(x, y) \in \mathbb{R}^{2} \mid y-a x-b>\delta\right\}$ and $D^{-}=\left\{(x, y) \in \mathbb{R}^{2} \mid y-a x-b<-\delta\right\}$, if $\alpha:[0,1] \rightarrow U \subset \mathbb{R}^{2}$ is a curve such that $\alpha(0) \in D^{+}$and $\alpha(1) \in D^{-}$, there exists an interval $\left[t_{0}, t_{1}\right] \subset[0,1]$, such that $\alpha\left(t_{0}\right) \in D \cap \partial D^{+}, \alpha\left(t_{1}\right) \in D \cap \partial D^{-}$and $\alpha(t) \in D, \forall t \in\left[t_{0}, t_{1}\right]$. Therefore,

$$
\ell(\alpha) \geq \int_{t_{0}}^{t_{1}} \widetilde{\varphi}(\alpha(t))\left|\alpha^{\prime}(t)\right| d t \geq \int_{t_{0}}^{t_{1}} r\left|\alpha^{\prime}(t)\right| d t \geq r \int_{t_{0}}^{t_{1}}\left|\alpha^{\prime}(t)\right| d t \geq r \frac{2 \delta}{\sqrt{1+a^{2}}} .
$$

Theorem 4.6 Any surface locally associated to the helicoid by a Ribaucour transformation as in Proposition 4.1 is complete.

Proof. We will exclude from our proof the helicoid, which is complete. The other surfaces locally associated to the helicoid by a Ribaucour transformation are given by (3.17), whose first fundamental form is $d s^{2}=\widetilde{\varphi}^{2} d z d \bar{z}$ given by (3.19), where

$$
\varphi=\cosh (\operatorname{Re} \sqrt{i} z)
$$

$F$ and $\beta$ are given by (4.1) and (4.2) respectively. Hence

$$
\begin{aligned}
\widetilde{\varphi} & =\frac{\varphi}{2|c|}\left[4\left|\frac{F_{z}}{F}-\frac{\sqrt{2}(1+i)}{4} \tanh (\operatorname{Re} \sqrt{i z})\right|^{2}+\frac{1}{\varphi^{2}}\right] \\
& =\frac{\varphi}{2|c|}\left[4\left|\frac{F_{z}}{F}\right|^{2}-\frac{2 \sqrt{2}\left(\operatorname{Re} F_{z}+\operatorname{Im} F_{z}\right)}{F} \tanh (\operatorname{Re} \sqrt{i} z)+1\right] \\
& \geq \frac{\varphi}{2|c|}\left[4\left|\frac{F_{z}}{F}\right|^{2}-\frac{2 \sqrt{2}\left(\operatorname{Re} F_{z}+\operatorname{Im} F_{z}\right)}{F}+1\right] .
\end{aligned}
$$


Since $\beta$ is given by (4.2) we have that

$$
\beta=\frac{\rho}{\sqrt{2}}\left(m+\frac{i}{m}\right), \quad \rho= \pm 1 .
$$

where $m$ is given by (4.4). We introduce the following notation

$$
\begin{aligned}
H & =\cosh (\operatorname{Re} \beta z+\mu)-\sigma \sin (\operatorname{Im} \beta z+\nu) \\
\theta_{ \pm} & =m \pm \frac{1}{m}
\end{aligned}
$$

From (4.1), (4.9) and (4.11) we have that

$$
\begin{aligned}
\left|\left(\operatorname{Re} F_{z}+\operatorname{Im} F_{z}\right)\right| & =\frac{1}{2 \sqrt{2}}\left|\sigma\left(\theta_{+}\right) \sinh (\operatorname{Re} \beta z+\mu)-\left(\theta_{-}\right) \cos (\operatorname{Im} \beta z+\nu)\right| \\
& \leq \frac{1}{2 \sqrt{2}}\left[\left(\theta_{+}\right) \cosh (\operatorname{Re} \beta z+\mu)+\left|\theta_{-}\right||\cos (\operatorname{Im} \beta z+\nu)|\right]
\end{aligned}
$$

thus

$\widetilde{\varphi} \geq \frac{\varphi}{|c|}\left[\frac{H}{4 \sigma F}\left(\theta_{+}^{2}-2\right)+\frac{1}{2}-\frac{\sigma \theta_{+}}{2 \sigma F} \cosh (\operatorname{Re} \beta z+\mu)-\frac{\left|\theta_{-}\right|}{2 \sigma F}|\cos (\operatorname{Im} \beta z+\nu)|\right]$

Claim 1 There are real numbers $r_{1}>0$ and $\delta_{1}>0$ such that $\widetilde{\varphi}(z) \geq r_{1}, \forall z \in \mathbb{C} \backslash T$, where

$$
T=\left\{z \in \mathbb{C} \mid-\delta_{1}<\operatorname{Re} \beta z+\mu<\delta_{1}\right\} .
$$

Indeed, consider $\varepsilon_{1}$ such that

$$
\varepsilon_{1}>\max \left\{0, \frac{4\left(\theta_{+}-2 \sigma-\theta_{-}\right)}{\theta_{+}^{2}-2 \sigma \theta_{+}}\right\} .
$$

Consider also $\delta_{1}>0$ given by

$$
\cosh \left(\delta_{1}\right)=1+\varepsilon_{1}
$$

and $T$ the region of the complex plane limited by the lines $\operatorname{Re} \beta z+\mu= \pm \delta_{1}$, i.e. $T$ given by (4.13). If $z \notin T$ we have that

$$
\cosh (\operatorname{Re} \beta z+\mu) \geq \cosh \left(\delta_{1}\right)=1+\varepsilon_{1},
$$

hence, from (4.12) we get

$$
|c| \frac{\widetilde{\varphi}}{\varphi} \geq \frac{H}{4 \sigma F}\left(\theta_{+}^{2}-2\right)+\frac{1}{2}-\frac{\sigma \theta_{+}}{2 F} \cosh (\operatorname{Re} \beta z+\mu)-\frac{\sigma\left|\theta_{-}\right|}{2 F} .
$$

It follows from (4.1) that

$$
|c| \frac{\widetilde{\varphi}}{\varphi} \geq \frac{H}{4 \sigma F}\left[\theta_{+}^{2}-\sigma \theta_{+}-2\right]+\frac{1}{2}-\frac{\sigma \theta_{+}}{4}-\frac{\sigma\left|\theta_{-}\right|}{2 F} .
$$


Recall from (4.4) that $m>0$ and $m \neq 1$. Moreover, the expression above is invariant under the transformation $m \rightarrow \frac{1}{m}$. In fact, this transformation keeps $\theta_{+}$invariant and it changes the sign of $\theta_{-}$. Hence, we only need to consider $0<m<1$, i.e., $0<\theta_{+}+\theta_{-}<2$. Observe also that for these values of $m$, we have $\theta_{+}>2$ and $\theta_{-}<0$.

From (4.10) we get

$$
\begin{aligned}
|c| \frac{\widetilde{\varphi}}{\varphi} & \geq \frac{H}{4 \sigma F}\left(\theta_{+}^{2}-\sigma \theta_{+}-2\right)+\frac{1}{2}-\frac{\sigma \theta_{+}}{4}+\frac{\sigma \theta_{-}}{2 F} \\
& \geq \frac{H}{4 \sigma F}\left(\theta_{+}^{2}-2 \sigma \theta_{+}\right)+\frac{\sigma \theta_{-}}{2 F}-\frac{\sigma \theta_{+}-2}{2 F} \sin (\operatorname{Im} \beta z+\nu)
\end{aligned}
$$

Thus

$$
|c| \frac{\widetilde{\varphi}}{\varphi} \geq \frac{1}{4 \sigma F}\left[H\left(\theta_{+}^{2}-2 \sigma \theta_{+}\right)+2\left(2 \sigma-\theta_{+}+\theta_{-}\right)\right] .
$$

Since $z \in T$, it follows from (4.16) and (4.10) that

$$
H\left(\theta_{+}^{2}-2 \sigma \theta_{+}\right) \geq \varepsilon_{1}\left(\theta_{+}^{2}-2 \sigma \theta_{+}\right) .
$$

Hence,

$$
|c| \frac{\widetilde{\varphi}}{\varphi} \geq \frac{1}{4 \sigma F}\left[H \frac{\theta_{+}^{2}-2 \sigma \theta_{+}}{2}+\frac{\varepsilon_{1}\left(\theta_{+}^{2}-2 \sigma \theta_{+}\right)}{2}+2\left(2 \sigma-\theta_{+}+\theta_{-}\right)\right] .
$$

As a consequence of (4.14), $\varepsilon_{1}$ satisfies the relation

$$
\frac{\varepsilon_{1}\left(\theta_{+}^{2}-2 \sigma \theta_{+}\right)}{2}+2\left(2 \sigma-\theta_{+}+\theta_{-}\right)>0 .
$$

Therefore, it follows from (4.10) that

$$
\frac{\widetilde{\varphi}}{\varphi} \geq \frac{(\cosh (\operatorname{Re} \beta z+\mu)-1)\left(\theta_{+}^{2}-2 \sigma \theta_{+}\right)}{8 \sigma|c| F} .
$$

From (4.16) we have

$$
1-\frac{1}{\cosh (\operatorname{Re} \beta z+\mu)} \geq 1-\frac{1}{1+\varepsilon_{1}}=\frac{\varepsilon_{1}}{1+\varepsilon_{1}} .
$$

Thus,

$$
\frac{\widetilde{\varphi}}{\varphi} \geq \frac{\theta_{+}^{2}-2 \sigma \theta_{+}}{16|c|}\left(1-\frac{1}{\cosh (\operatorname{Re} \beta z+\mu)}\right) \geq \frac{\left(\theta_{+}^{2}-2 \sigma \theta_{+}\right) \varepsilon_{1}}{16|c|\left(1+\varepsilon_{1}\right)}=r_{1}>0 .
$$

Hence, we get $\widetilde{\varphi} \geq r_{1}$.

Claim 2 There are real numbers $r_{2}>0$ and $\delta_{2}>0$ such that $\widetilde{\varphi}(z) \geq r_{2}, \forall z \in T \cap D$, where $D=\bigcup_{k \in \mathbb{Z}} D_{k}$ with

$$
D_{k}=\left\{z \in \mathbb{C} \mid-\delta_{2}<\operatorname{Im} \beta z+\nu+\frac{\sigma \pi}{2}-2 k \pi<\delta_{2}\right\}
$$


In fact, let $z_{k} \in \mathbb{C}$ be a planar end of $\widetilde{X}$. Then $F\left(z_{k}, \bar{z}_{k}\right)=0$ i.e., $\cosh \left(\operatorname{Re} \beta z+\mu_{k}\right)=1$ and $\sin \left(\operatorname{Im} \beta z+\nu_{k}\right)=-\sigma$. Then $z_{k}$ belongs to the lines $\operatorname{Re} \beta z+\mu=0$ and $\operatorname{Im} \beta z+\nu=-\sigma \frac{\pi}{2}+2 k \pi$ which are orthogonal (see Remark 4.3). Consider $\varepsilon_{2} \in \mathbb{R}$, such that

$$
0<\varepsilon_{2}<\min \left\{1, \frac{\theta_{+}^{2}-2 \theta_{+}}{4\left|\theta_{-}\right|}\right\} .
$$

Let $\delta_{2}$ be a real number such that $0<\delta_{2}<\pi / 2, \sin \delta_{2}=\varepsilon_{2}$ and let $D_{k}$ be a region of the complex plane bounded by the lines $\operatorname{Im} \beta z+\nu=-\frac{\sigma \pi}{2}+2 k \pi \pm \delta_{2}$, i.e., $D_{k}$ given by (4.17). If $z \in D_{k}$, then $\operatorname{Im} \beta z+\nu \in\left(-\frac{\sigma \pi}{2}+2 k \pi-\delta_{2},-\frac{\sigma \pi}{2}+2 k \pi+\delta_{2}\right)$.

If $\sigma= \pm 1$, we get

$$
\cos \left(-\frac{\sigma \pi}{2}+2 k \pi \mp \delta_{2}\right)<\cos (\operatorname{Im} \beta z+\nu)<\cos \left(-\frac{\sigma \pi}{2}+2 k \pi \pm \delta_{2}\right)
$$

which implies that

$$
-\sin \delta_{2}<\cos (\operatorname{Im} \beta z+\nu)<\sin \delta_{2} .
$$

Therefore, for any value of $\sigma$ we get $|\cos (\operatorname{Im} \beta z+\nu)|<\varepsilon_{2}$ and $\sigma \sin (\operatorname{Im} \beta z+\nu)<0$. It follows from (4.12) that

$$
|c| \frac{\widetilde{\varphi}}{\varphi} \geq \frac{H}{4 \sigma F}\left(\theta_{+}^{2}-2\right)+\frac{1}{2}-\frac{\theta_{+}}{2 \sigma F} \cosh (\operatorname{Re} \beta z+\mu)-\frac{\left|\theta_{-}\right|}{2 \sigma F}|\cos (\operatorname{Im} \beta z+\nu)| .
$$

Using (4.10) we have

$$
\begin{aligned}
|c| \frac{\widetilde{\varphi}}{\varphi} \geq & \frac{H}{4 \sigma F}\left(\theta_{+}^{2}-2\right)+\frac{1}{2}-\frac{\theta_{+}}{2 \sigma F} \cosh (\operatorname{Re} \beta z+\mu)-\frac{\left|\theta_{-}\right|}{2 \sigma F}|\cos (\operatorname{Im} \beta z+\nu)| \\
\geq & \frac{1}{4 \sigma F}\left(\theta_{+}^{2}-2 \theta_{+}-2\right) \cosh (\operatorname{Re} \beta z+\mu)-\frac{\sigma}{4 \sigma F}\left(\theta_{+}^{2}-2\right) \sin (\operatorname{Im} \beta z+\nu)+ \\
& +\frac{1}{2}-\frac{\left|\theta_{-}\right|}{2 \sigma F}|\cos (\operatorname{Im} \beta z+\nu)| .
\end{aligned}
$$

However,

$$
\frac{\sigma}{4 \sigma F}\left(4-\theta_{+}^{2}\right) \sin (\operatorname{Im} \beta z+\nu)>0,
$$

since $\theta_{+}>2$ and $\sigma \sin (\operatorname{Im} \beta z+\nu)<0$

$$
\frac{\widetilde{\varphi}}{\varphi} \geq \frac{1}{4|c| \sigma F}\left[\theta_{+}^{2}-2 \theta_{+}-2\left|\theta_{-}\right||\cos (\operatorname{Im} \beta z+\nu)|\right] .
$$

Since $z \in D_{k}$, then $|\cos (\operatorname{Im} \beta z+\nu)|<\varepsilon_{2}$, and

$$
\frac{\widetilde{\varphi}}{\varphi} \geq \frac{1}{4|c| \sigma F}\left[\theta_{+}^{2}-2 \theta_{+}-2\left|\theta_{-}\right| \varepsilon_{2}\right] .
$$

From (4.18), we have that

$$
\frac{\theta_{+}^{2}-2 \theta_{+}}{2}-2\left|\theta_{-}\right| \varepsilon_{2}>0
$$


Since $z \in T$ we get $\sigma F \leq 2 \cosh (\operatorname{Re} \beta z+\mu)<2 \cosh \left(\delta_{1}\right)=2\left(1+\varepsilon_{1}\right)$, from (4.15). It follows from (4.20) that

$$
\frac{\widetilde{\varphi}}{\varphi} \geq \frac{\theta_{+}^{2}-2 \theta_{+}}{32|c|\left(1+\varepsilon_{1}\right)}=r_{2}>0
$$

hence $\widetilde{\varphi} \geq r_{2}>0$.

Claim 3 Any divergent curve on the surface $\widetilde{X}$ given by (3.17) has infinite length.

Indeed, let $\alpha(t)=(x(t), y(t))$ be a divergent curve such that $\lim _{t \rightarrow \infty} \alpha(t)=z_{k}$, for some $k \in \mathbb{Z}$. Then the length of $\widetilde{X}(\alpha)$ is infinite, as a consequence of Theorem 1.4.

If $\alpha(t)=(x(t), y(t)), t \in[0, \infty)$ is a regular curve such that $x^{2}+y^{2} \rightarrow \infty$, when $t \rightarrow \infty$, then the length $\ell(\widetilde{X}(\alpha(t)))=\infty$. In fact, consider $r_{1}, r_{2}, \delta_{1}, \delta_{2}$ given by Claim 1 and Claim 2 and $r=\min \left\{r_{1}, r_{2}\right\}$. If there exists $t_{1}>0$ such that $\forall t \geq t_{1}, \alpha(t) \notin T$, then it follows from Claim 1 that $\forall t \geq t_{1}$ we get $\widetilde{\varphi}(\alpha(t)) \geq r$, thus

$$
\ell\left(\widetilde{X}_{c}(\alpha(t))\right) \geq \int_{t_{1}}^{\infty} r d t=\infty .
$$

On the other hand, if $\forall t_{1}$, there exists $t \geq t_{1}$ such that $\alpha(t) \in T$, since $x^{2}+y^{2} \rightarrow \infty$, when $t \rightarrow \infty$, the curve $\alpha$ crosses an infinite number of strips $D_{k}$. But, by Claim 1 , we get $\widetilde{\varphi} \geq r$ in $D_{k}$ for all $k \in \mathbb{Z}$, since the width of each strip $D_{k}$, is $2 \delta_{2} m^{2} / \sqrt{1+m^{4}}$, we conclude the proof of Claim 3, by using Lemma 4.5. This completes the proof of Theorem 4.6.

Proposition 4.7 Consider the helicoid with the Weierstrass data $g(z)=-i e^{\sqrt{i z}}$, $f=1 / g^{\prime}$ and the corresponding parametrization $X(z, \bar{z})$. Excluding the helicoid, any surface of the family $\widetilde{X}(z, \bar{z})$ locally associated to $X$ by a Ribaucour transformation as in Proposition 4.1, has a Weierstrass data $\tilde{g}$ given by (3.18) and $\widetilde{f}=1 / \widetilde{g}^{\prime}$, where $\varphi=$ $\cosh (\operatorname{Re} \sqrt{i} z)$ and $F(z, \bar{z})$ is given by $(4.1)$.

Proof. The result is a consequence of Theorem 3.4.

In [CFT2], one can find more details on the minimal surfaces associated to the catenoid and to Enneper's surface. As an application of section 3, we will present a Weierstrass representation of these surfaces.

Proposition 4.8 Let $X(z, \bar{z})$ be a parametrization of Enneper's surface given by the Weierstrass data $g(z)=z$ and $f=1 / g^{\prime}$. Excluding Enneper's surface, any surface of the family $\widetilde{X}(z, \bar{z})$, locally associated to $X$ by a Ribaucour transformation as in Theorem 3.4, has Weierstrass data $\tilde{g}$ given by (3.18) and $\widetilde{f}=1 / \widetilde{g}^{\prime}$, where $\varphi=(1+z \bar{z}) / 2$ and

$$
F(z, \bar{z})=\sigma \cosh (\operatorname{Re} \rho \sqrt{-2 c} z+\mu)+\sin (\operatorname{Im} \rho \sqrt{-2 c} z+\nu)
$$

where $c \neq 0$ is a real number, and $\mu, \nu \in \mathbb{R}, \sigma, \rho= \pm 1$. 
Proof. The result is a consequence of Theorem 3.4.

Proposition 4.9 Let $X(z, \bar{z})$ be a parametrization of the catenoid given by the Weierstrass data $g(z)=-e^{z}$ and $f=1 / g^{\prime}$. Excluding the catenoid, any surface of the family $\widetilde{X}(z, \bar{z})$, locally associated to $X$ by a Ribaucour transformation as in Theorem 3.4, has Weierstrass data $\tilde{g}$ given by (3.18) and $\tilde{f}=1 / \widetilde{g}^{\prime}$, where $\varphi=\cosh (\operatorname{Re} z)$ and

$$
F(z, \bar{z})=\sigma \cosh (\operatorname{Re} \rho \sqrt{1-2 c} z+\mu)+\sin (\operatorname{Im} \rho \sqrt{1-2 c} z+\nu)
$$

with $\mu, \nu \in \mathbb{R}, \sigma, \rho= \pm 1$ if $c \neq 1 / 2$ and

$$
F(z, \bar{z})=b_{1} z \bar{z}+b_{3} z+\bar{b}_{3} \bar{z}+b_{2}
$$

with $b_{1} \neq 0, b_{2} \in \mathbb{R}, b_{3} \in \mathbb{C}$ and $b_{1} b_{2}=\left|b_{3}\right|^{2}$ if $c=1 / 2$.

Proof. The result is a consequence of Theorem 3.4.

\section{References}

[Bi] L. Bianchi. Le transformazioni di Ribaucour dei sistemi ortogonali e il teorema generale di permutabilitá. Annali di Matematica (3), 27(1918), 183-253 and (3), 28(1919), 187-233.

[CFT1] A. V. Corro, W. Ferreira, and K. Tenenblat. On Ribaucour transformation for hypersurfaces. Mat. Comtemp. 17 (1999), 137-160.

[CFT2] A. V. Corro, W. Ferreira, and K. Tenenblat. Minimal surfaces obtained by Ribaucour transformation. Geometriae Dedicata 96 (2003), 177-150.

[CFT3] A. V. Corro, W. Ferreira, and K. Tenenblat. Ribaucour transformation for constant mean curvature and linear Weingarten surfaces. Pacific Journal of Mathematics, vol. 212 (2003), 256-296.

[CHM] M. Callahan, D. Hoffman, and W. Meeks III. Embedded minimal surfaces with an infinite numbers of ends. Invent. Maths., 96 (1989), 459-505.

[CT] A. V. Corro and K. Tenenblat. Ribaucour transformations revisited. Comm. Anal. Geom., 12 (2004), 1055-1082.

[Co] C. J. Costa. Example of a complete minimal immersion in $\mathbb{R}^{3}$ of genus one and three embedded ends. Bull. Soc. Bras. Mat. 15 (1984), 47-54.

[HM] D. Hoffman and W. Meeks III. Embedded minimal surfaces of finite topology. Ann. of Math. 131 (1990), 1-34.

[HU] A. Huber. On subharmonic functions and differential geometry in the large. Comment. Math. Helv. 32 (1957), 13-72. 
[JM] L. P. Jorge and W. Meeks III. The topology of complete minimal surfaces of finite total Gaussiann curvature. Topology 2 (1983), 203-221.

[W] Q. Wang. Transformações de Ribaucour para hipersuperfícies na esfera e no espaço hiperbólico. Ph.D. thesis, UnB (2004).

M. V. Lemes

Instituto de Matemática e Estatística

Universidade Federal de Goiás

74001-970, Goiânia, GO, Brazil

max@mat.ufg.br
K. Tenenblat

Departamento de Matemática, Universidade de Brasília 70904-970, Brasília, DF, Brazil

keti@mat.unb.br 